\title{
Funcionalidad física y Calidad de vida en Mujeres con Sobrepeso y Obesidad
}

\author{
Dennys Leonardo Abril Merizalde, $\mathrm{MsC}$ \\ Catherine Alexandra Andrade Trujillo, $\mathrm{MsC}$ \\ Docente Escuela Superior Politécnica de Chimborazo, \\ ESPOCH, Riobamba, Ecuador
}

Patricia del Carmen Chico López, MsC

Docente de Carrera Nutrición y Dietética, Escuela Superior Politécnica de Chimborazo ESPOCH, Vicedirectora del Instituto de Posgrado y Educación Continua (IPEC), Riobamba, Ecuador

Eulalia Teresita Santillán Mancero, $\mathrm{MgS}$

Directora y Docente de Carrera Nutrición y Dietética, Escuela Superior

Politécnica de Chimborazo, ESPOCH, Riobamba, Ecuador

Lilia Ofir Peralta Saá, MgS

Docente de Carrera Nutrición y Dietética, Escuela Superior Politécnica de

Chimborazo, ESPOCH, Riobamba, Ecuador

Doi: 10.19044/esj.2019.v15n3p143～URL:http://dx.doi.org/10.19044/esj.2019.v15n3p143

\section{Resumen}

La obesidad y el sobrepeso consisten en una excesiva acumulación de grasa corporal en el organismo, este exceso afecta la funcionalidad musculoesquelética predisponiendo a desarrollar afecciones biomecánicas particularmente de la extremidad inferior disminuyendo la capacidad funcional del individuo. El presente estudio tiene como objetivo: analizar la relación entre el salto vertical y la calidad de vida en mujeres con sobrepeso y obesidad. Se incluyó 78 mujeres adultas con sobrepeso y obesidad, seleccionadas de una base de datos establecida perteneciente al Programa de Control de Peso de la Unidad de Medicina del Deporte de la UNAM. La capacidad funcional fue medida mediante el salto vertical, una prueba de función física fácil que indica la función del tren inferior. La calidad de vida fue evaluada mediante el Impact Weight Quality of Life (IWQOL) un cuestionario de fácil aplicación, confiable, reproducible, con alta validez interna y sensible para detectar diferencias entre los distintos grados de obesidad. Al analizar el salto vertical se observó que mantiene una relación positiva con el puntaje total del cuestionario IWQOL ( $r=0.229$; $p=0.044)$; es decir, a medida que aumenta el salto vertical aumenta el puntaje total del cuestionario IWQOL; de igual manera, se evidencia una relación positiva 
entre los rubros del cuestionario IWQOL y el salto vertical: actividades de la vida diaria $(\mathrm{r}=0.390 ; \mathrm{p}=0.000)$ y actividad física $(\mathrm{r}=-0.270 ; \mathrm{p}=0.017)$. Se concluye que el salto vertical se relaciona positivamente con la calidad de vida, además los indicadores diagnósticos de obesidad influyen negativamente en el salto vertical y la calidad de vida.

Palabras clave: Función física, calidad de vida, sobrepeso, obesidad

\title{
Physical Functionality and Quality of Life in Overweight and Obesity Women
}

\section{Dennys Leonardo Abril Merizalde, MsC}

Catherine Alexandra Andrade Trujillo, $\mathrm{MsC}$

Docente Escuela Superior Politécnica de Chimborazo,

ESPOCH, Riobamba, Ecuador

\section{Patricia del Carmen Chico López, $\mathrm{MsC}$}

Docente de Carrera Nutrición y Dietética, Escuela Superior Politécnica de Chimborazo ESPOCH, Vicedirectora del Instituto de Posgrado y Educación

Continua (IPEC), Riobamba, Ecuador

\section{Eulalia Teresita Santillán Mancero, $\mathrm{MgS}$}

Directora y Docente de Carrera Nutrición y Dietética, Escuela Superior

Politécnica de Chimborazo, ESPOCH, Riobamba, Ecuador

\section{Lilia Ofir Peralta Saá, MgS}

Docente de Carrera Nutrición y Dietética, Escuela Superior Politécnica de

Chimborazo, ESPOCH, Riobamba, Ecuador

\begin{abstract}
Obesity and overweight consist in an excessive accumulation of body fat in the organism, this excess affects the muscular functionality that predisposes to the development of biomechanical affections in particular of the lower limb that diminish the functional capacity of an individual. The present study aims to: analyze the relationship between vertical jump and quality of life in overweight and obese women. We included 78 adult women with overweight and obesity, selected from a database belonging to the Weight Control Program of the Sports Medicine Unit of the UNAM. The functional capacity was measured by the vertical jump, an easy physical function test that indicates the function of the lower train. Quality of life was achieved through
\end{abstract}


the impact of quality of life (IWQOL) a questionnaire of easy application, reliable, reproducible, with high internal validity and sensitive for different degrees of obesity. When analyzing the vertical jump, a positive relation was observed with the total score of the IWQOL questionnaire $(\mathrm{r}=0.229, \mathrm{p}=$ 0.044); that is, a measure that increases the vertical jump increases the total score of the IWQOL questionnaire, likewise a positive relationship between the items of the IWQOL questionnaire and the vertical jump is shown: activities of daily living $(\mathrm{r}=0.390, \mathrm{p}=0.000)$ and physical activity $(\mathrm{r}=$ $0.270, p=0.017$ ). It is concluded that the vertical jump is positively related to the quality of life, in addition the diagnostic indicators of obesity negatively influence the vertical jump and the quality of life.

Keywords: Physical function, quality of life, overweight, obesity

\section{Introducción}

La obesidad en México es un problema de salud pública que se ha incrementado en el periodo de 1988 al 2012, el sobrepeso en mujeres de 20 a 49 años de edad incrementó de 25 a $35.3 \%$ y la obesidad de 9.5 a $35.2 \%$, mientras que la prevalencia combinada de sobrepeso y obesidad (IMC $\geq 25$ $\mathrm{kg} / \mathrm{m} 2$ ) es mayor en las mujeres (73.0\%) que en los hombres $(69.4 \%)$. (ENSANUT, 2012). La obesidad y el sobrepeso consisten en una excesiva acumulación de grasa corporal en el organismo, producto de la interacción de una serie de factores genéticos, ambientales y de estilo de vida, que influyen en provocar un desequilibrio entre la ingesta calórica y el gasto energético (Navara, 2011) (Garawi, 2014) (William, 2009).

El exceso de grasa corporal afecta la funcionalidad musculoesquelética del individuo y lo predisponen a desarrollar afecciones biomecánicas particularmente de la extremidad inferior (cadera, rodilla, tobillo y pie) (Feldman, 2002) (León, 2012) (Barrios, 2010), estas afecciones tienen un impacto sobre la marcha y el hueso, predisponiendo el desarrollo de trastornos articulares, tales como fracturas, osteoartritis, fascitis plantar y lumbalgia (Tomaselli, 2001) (Lovejoy, 2003), a medida que incrementa el IMC $\left(\geq 40 \mathrm{~kg} / \mathrm{m}^{2}\right)$ existe una afección de los músculos extensores de la rodilla (cuádriceps), disminuyendo la capacidad funcional del individuo ( $-33 \%$ en hombres, -37\% en mujeres) (Picavet, 2003) que lo predispone a desarrollar hábitos sedentarios que aumentan el riesgo de padecer comorbilidades asociadas a la obesidad (diabetes mellitus tipo II, hipertensión arterial, dislipidemias, enfermedades cardiovasculares) (Dakin, 2014) (Michele, 2012) (Franceschi, 2014).

A medida que incrementa la edad, un individuo disminuye su masa muscular y su fuerza (15\% por década después de los 50 años que puede llegar hasta $30 \%$ por década después de los 70 años) esto desencadena una serie de 
afecciones como discapacidad física y mortalidad, (Gonelli, 2014) (NIH, 2001); sin embargo, la fuerza muscular es un factor protector contra el desarrollo de síndrome metabólico independiente del peso y la edad (44\% menos riesgo), (Alhambra, 2012) mientras que la disminución en la fuerza muscular está relacionada con muerte por enfermedad cardiovascular, siendo $137 \%$ más probable presentar en hombres de 40 a 84 años comparado con individuos que realizan entrenamiento de fuerza muscular. (Reyna, 2013) (Fredman, 2012). Existe varias pruebas que miden fuerza muscular, entre las que miden fuerza del tren inferior está el salto vertical, es una prueba fácil y práctica que se ha utilizado poco en el ámbito clínico e indica la función de todo un segmento corporal (tren inferior), está asociada con el desempeño físico en atletas y lo relacionan como un indicador de fuerza rápida del tren inferior (Cámara, 2011) (Contreras, 2011).

La Organización Mundial de la salud (OMS, 2005) define a la cálida de vida como "la percepción que un individuo tiene de su lugar en la existencia, en el contexto de la cultura y del sistema de valores en los que vive y en relación con sus objetivos, sus expectativas, sus normas, sus inquietudes." La calidad de vida en personas con sobrepeso o algún grado de obesidad se ve influenciada negativamente, a mayor sea el IMC, disminuye su calidad de vida (evidenciado por cuestionario Impact Weight Quality Of Life), comparada con persona de peso normal, siendo más evidente el deterioro en mujeres con IMC $>30 \mathrm{~kg} / \mathrm{m}^{2}$ (Crespo, 1996).

Uno de los instrumentos que evalúan calidad de vida es el Impact Weight Quality Of Life (IWQOL) es un cuestionario desarrollado específicamente para evaluar los efectos del peso sobre la calidad de vida del sujeto con obesidad, incluye ocho rubros: salud, alimentación, socialinterpersonal, vida sexual, autoestima, actividades de la vida diaria, trabajo, y actividad física. En la población mexicana se validó el instrumento, donde se determinó la reproducibilidad y sensibilidad del mismo, determinando que el cuestionario "es confiable para investigación de la calidad de vida en mexicanos y probablemente en la mayoría de las poblaciones de Latinoamérica." (Bolado, 2008). El cuestionario incluye ocho dominios: salud, alimentación, social-interpersonal, vida sexual, autoestima, actividades de la vida diaria, trabajo y actividad física, cada dominio es variable en un número de preguntas entre 6 y 14 para un total de 72 . Fue empleada una escala de Likert en cada pregunta: siempre, frecuentemente, a veces, rara vez y nunca, opciones a las cuales se les adjudicaron valores de 1, 2, 3, 4, y 5 respectivamente. Así la cuenta total máxima es 360. Partiendo de este contexto donde la obesidad tiene mayor prevalencia en mujeres, y se evidencia afecciones musculoesqueléticas que aumentan con la edad, se plantea utilizar el salto vertical como medida de función musculoesquelética (fuerza muscular del tren inferior) y su posible relación con la calidad de vida mediante el 
cuestionario Impact Weight Quality Of Life (IWQOL) en mujeres adultas con sobrepeso y obesidad (William, 2009).

En torno a esta problemática, el objetivo de la presente investigación fue analizar la relación entre el salto vertical y la calidad de vida en mujeres con sobrepeso y obesidad.

\section{Metodología}

El presente es un estudio transversal, retrolectivo, analítico. Para la obtención de la muestra se utilizó un método de muestreo no probabilístico, muestreo por conveniencia, las participantes candidatas al Programa de Control de Peso de la Unidad de Medicina del Deporte de la UNAM fueron reclutadas mediante convocatoria abierta, para el presente estudio se partió de una base de datos ya capturada de 290 participantes, de la cual se seleccionó aquellas que cumplieron con los criterios de inclusión planteados para la presente investigación, la muestra está conformada por 78 pacientes mujeres.

Criterios de inclusión: Sexo femenino, edad entre 18 a 75 años, Con sobrepeso y obesidad (IMC $\geq 25 \mathrm{~kg} / \mathrm{m}^{2}$ ), con cualquier nivel de actividad física. Criterios de exclusión: Osteoartritis severa, cirugías recientes, lesiones agudas, tratamiento fisioterapéutico reciente.

Para la medición del salto vertical el equipo requerido es una superficie plana con saltímetro (mide la diferencia en centímetros entre la medida registrada del individuo parado con la mano extendida y el mejor salto en tres intentos). El sujeto se coloca con las piernas flexionadas por la rodilla en un ángulo de $90^{\circ}$, después de mantener la posición, da un salto lo más alto posible en toda su extensión para llegar a un disco, evitando cualquier acción de contra movimiento y sin soltar las manos, cayendo en la misma posición con los pies y las piernas extendidas. (Nahdiya-Zainal, 2013). El resultado se interpreta mediante el salto relativo ( $\mathrm{cm}$ de diferencia de salto/kg de peso), con la finalidad de homologar la relación del peso corporal con la fuerza muscular medida mediante el salto vertical.

El cuestionario IWQOL es auto-administrado e incluye ocho dominios: salud, alimentación, social-interpersonal, vida sexual, autoestima, actividades de la vida diaria, trabajo y actividad física, con 72 dominios en total. Emplea para la valoración una escala tipo Likert: siempre, frecuentemente, a veces, rara vez y nunca, opciones a las cuales se les adjudican valores de $1,2,3,4$, y 5 respectivamente, para un máximo total de 360 puntos.

\section{Resultados}

La muestra total está conformada por 78 mujeres, la media de edad es $40.96 \pm 11.97$ años, según el IMC $(31.81 \pm 4.73 \mathrm{Kg} / \mathrm{m} 2)$ la población en estudio presenta obesidad grado I, de acuerdo al porcentaje de grasa la media 
es de $45.77 \pm 5.04$ colocando al grupo en estudio en obesidad, según el porcentaje de musculo la media general es de $24.52 \pm 3.62$ y según la circunferencia de cintura la media global es de $98.73 \pm 11.90$ colocando a la muestra en obesidad abdominal. La media global de salto relativo es $0.167 \pm$ $0.067 \mathrm{~cm} / \mathrm{kg}$ mientras que la media de la puntuación global del cuestionario IWQOL es de 212.10 \pm 33.27 puntos, siendo el rubro de salud el más alto con una media de $42.74 \pm 6.30$ puntos, y el rubro de vida sexual el más bajo con una media de $15.92 \pm 5.33$ puntos. Variables descritas en la tabla 1 .

Tabla 1: Características generales de la muestra.

\begin{tabular}{|l|l|}
\hline Variable & Muestra total $(\mathbf{n}=\mathbf{7 8})$ Media \pm SD \\
\hline EDAD (ANOS) & $40.96 \pm 11.97$ \\
\hline PORCENTAJE DE GRASA (\%) & $45.77 \pm 5.04$ \\
\hline PORCENTAJE DE MUSCULO (\%) & $24.52 \pm 3.62$ \\
\hline CIRCUNFERENCIA CINTURA (cm) & $98.73 \pm 11.90$ \\
\hline IMC (kg/m ${ }^{2}$ ) & $31.81 \pm 4.73$ \\
\hline SALTO RELATIVO (cm/kg) & $0.167 \pm 0.067$ \\
\hline IWQOL TOTAL (Total) & $212.10 \pm 33.27$ \\
\hline R. SALUD & $42.74 \pm 6.30$ \\
\hline R. ALIMENTACION & $23.19 \pm 5.23$ \\
\hline R. SOCIAL-INTERPERSONAL & $36.19 \pm 8.57$ \\
\hline R. VIDA SEXUAL & $15.92 \pm 5.33$ \\
\hline R. AUTOESTIMA & $20.61 \pm 7.36$ \\
\hline R. ACTIVIDADES DE LA VIDA DIARIA & $20.79 \pm 5.07$ \\
\hline R. TRABAJO & $22.87 \pm 5.16$ \\
\hline R. ACTIVIDAD FISICA & $29.78 \pm 8.36$ \\
\hline $\begin{array}{l}\text { SD: desviación estándar; IMC: indice de masa corporal; IWQOL: Impact weight } \\
\text { quality of life; R: Rubro del cuestionario IWQL } *: \text { Frecuencia y porcentaje. }\end{array}$ \\
\hline
\end{tabular}

Tabla 2: Características generales de la muestra y análisis comparativo según grados de IMC (sobrepeso y obesidad).

\begin{tabular}{|c|c|c|c|c|}
\hline \multirow[b]{2}{*}{ Variable } & \multicolumn{4}{|c|}{ IMC $(n=78)$ Media \pm SD } \\
\hline & $\begin{array}{l}\text { Sobrepeso } \\
\leq 29.9 \mathrm{~kg} / \mathrm{m}^{2} \\
(\mathrm{n}=33)\end{array}$ & $\begin{array}{l}\text { Obesidad } \\
\geq 30 \mathrm{~kg} / \mathrm{m}^{2} \\
(\mathrm{n}=45)\end{array}$ & $t$ & $\bar{p}$ \\
\hline EDAD & $43.18 \pm 12.13$ & $39.33 \pm 11.71$ & 1.412 & 0.933 \\
\hline PORCENTAJE DE GRASA (\%) & $43.97 \pm 4.77$ & $47.06 \pm 4.83$ & -2.810 & 0.675 \\
\hline PORCENTAJE DE MUSCULO (\%) & $25.76 \pm 3.72$ & $23.56 \pm 3.25$ & 2.776 & 0.478 \\
\hline CIRCUNFERENCIA CINTURA $(\mathrm{cm})$ & $90.09 \pm 5.33$ & $105.06 \pm 11.41$ & -7.724 & $0.000^{\circ}$ \\
\hline SALTO RELATIVO $(\mathrm{cm} / \mathrm{kg})$ & $0.202 \pm 0.059$ & $0.142 \pm 0.061$ & 4.295 & 0.443 \\
\hline IWQOL (total) & $219.48 \pm 18.56$ & $206.68 \pm 40.19$ & 1.880 & $0.000^{\circ}$ \\
\hline R. SALUD & $43.42 \pm 5.03$ & $42.24 \pm 7.10$ & 0.815 & 0.288 \\
\hline R. ALIMENTACION & $22.75 \pm 5.92$ & $23.51 \pm 4.70$ & -0.626 & 0.340 \\
\hline R. SOCIAL-INTERPERSONAL & $37.70 \pm 6.70$ & $35.09 \pm 9.64$ & 1.408 & $0.002^{*}$ \\
\hline R. VIDA SEXUAL & $15.66 \pm 4.04$ & $16.11 \pm 6.14$ & -0.385 & $0.002^{*}$ \\
\hline R. AUTOESTIMA & $21.42 \pm 5.56$ & $20.02 \pm 8.46$ & 0.829 & 0.012 \\
\hline R. ACTIVIDADES DE LA VIDA DIARIA & $20.07 \pm 4.81$ & $21.51 \pm 5.29$ & 4.829 & $0.000^{\circ}$ \\
\hline R. TRABAJO & $23.48 \pm 4.98$ & $22.42 \pm 5.29$ & 0.897 & 0.851 \\
\hline R. ACTIVIDAD FISICA & $31.57 \pm 5.82$ & $28.44 \pm 9.67$ & 1.652 & 0.010 \\
\hline
\end{tabular}


De acuerdo con las características presentadas en la tabla 2, la población se concentra en los grupos de sobrepeso y obesidad grado I, de esta manera se dividió la muestra en dos grupos, sobrepeso $\leq 29.9 \mathrm{~kg} / \mathrm{m}^{2}(33$ participantes) y obesidad $\geq 30 \mathrm{~kg} / \mathrm{m}^{2}$ (45 participantes). De acuerdo con la circunferencia de cintura el grupo de obesidad tiene la media más alta (105.06 $\pm 11.41)$ en comparación con el grupo de sobrepeso $(90.09 \pm 5.33)$ siendo esta diferencia entre grupos estadísticamente significativa con un valor de $\mathrm{p}=$ 0.000. Según el puntaje total del IWQOL el grupo de sobrepeso presenta la mayor puntuación global $(219.48 \pm 18.56)$ en comparación con el grupo de obesidad (206.68 \pm 40.19), siendo esta diferencia entre grupos estadísticamente significativa con un valor de $\mathrm{p}=0.000$. Según el rubro social interpersonal el grupo de sobrepeso presenta la media más alta $(37.70 \pm 6.70)$ en comparación con el grupo de obesidad (35.09 \pm 9.64$)$, siendo esta diferencia entre grupos estadísticamente significativa con un valor de $p=0.002$. En el rubro vida sexual el grupo de obesidad presenta la media más alta (16.11 \pm 6.14) en comparación con el grupo de sobrepeso $(15.66 \pm 4.04)$, siendo esta diferencia entre grupos estadísticamente significativa con un valor de $\mathrm{p}=$ 0.002. En el rubro de actividades de la vida diaria el grupo de obesidad mantiene la media más alta $(21.51 \pm 5.29)$ en comparación con el grupo de sobrepeso $(20.07 \pm 4.81)$, siendo esta diferencia entre grupos estadísticamente significativa con un valor de $\mathrm{p}=0.000$.

Tabla 3: Correlación de variables según grados de IMC (Sobrepeso, Obesidad).

\begin{tabular}{|c|c|c|c|c|c|c|c|c|}
\hline \multicolumn{9}{|c|}{ CORRELACIONES SECON GRADOS DE IMC $(\mathrm{A}=78)$} \\
\hline $\begin{array}{l}\text { Grupos de } \\
\text { edad }\end{array}$ & Variables & & EOAD & $\begin{array}{l}\text { PORCENTAJE } \\
\text { DE GRASA (\%) }\end{array}$ & $\begin{array}{l}\text { PORCENTA } \\
\text { JE DE } \\
\text { MÚSCULO } \\
\text { (W) }\end{array}$ & $\begin{array}{l}\text { CIRCUNFER } \\
\text { ENCIA } \\
\text { CINTURA } \\
(\mathrm{cm})\end{array}$ & $\begin{array}{l}\text { SALTO } \\
\text { RELATIVO } \\
\text { (cm/kg) }\end{array}$ & $\begin{array}{l}\text { TWOOL } \\
\text { (puntale) }\end{array}$ \\
\hline \multirow{12}{*}{$\begin{array}{l}\text { Sobrepeso } \\
529.9 \\
\mathrm{~kg}^{2} \\
(n=39)\end{array}$} & \multirow[t]{2}{*}{ EDAD } & $t$ & 1 & 0.176 & -0.210 & 0.257 & $-0.407^{*}$ & 0.058 \\
\hline & & $p$ & & 0.327 & 0.240 & 0.148 & 0.019 & 0.750 \\
\hline & \multirow{2}{*}{$\begin{array}{l}\text { PORCENTAJE DE } \\
\text { GRASA (\%) }\end{array}$} & ? & 0.176 & 1 & $0.589^{* *}$ & 0.182 & 0.208 & -0.190 \\
\hline & & $p$ & 0.327 & & 0,000 & 0.300 & 0.245 & 0.200 \\
\hline & \multirow{2}{*}{$\begin{array}{l}\text { PORCENTAJE } \\
\text { MÚSCULO (\%) }\end{array}$} & $t$ & -0.210 & $0.589+4$ & 1 & $-0.490^{4 *}$ & $0.363^{*}$ & 0.315 \\
\hline & & p & 0.240 & 0.000 & & 0.004 & 0.035 & 0.074 \\
\hline & \multirow{2}{*}{$\begin{array}{l}\text { CIRCUNFERENCLA } \\
\text { CENTURA }(\mathrm{cm})\end{array}$} & $t$ & 0.257 & 0.182 & $-0.499^{* *}$ & 1 & $0.531 *$ & $-0.345^{\circ}$ \\
\hline & & $p$ & 0.148 & 0.309 & 0.004 & & 0.001 & 0049 \\
\hline & \multirow{2}{*}{$\begin{array}{l}\text { SALTO RELATIVO } \\
\text { (cm/kg) }\end{array}$} & $t$ & $-0.407^{\circ}$ & 0.208 & $0.365^{\circ}$ & $0.531 *$ & 1 & 0.128 \\
\hline & & P & 0.019 & 0.245 & 0.035 & 0.001 & & 0.477 \\
\hline & \multirow[t]{2}{*}{ IWGOL (puntaje) } & $r$ & 0.058 & -0.190 & 0.315 & $-0.345^{\circ}$ & 0.128 & 1 \\
\hline & & $p$ & 0.750 & 0.290 & 0.074 & 0.049 & 0.477 & \\
\hline \multirow{12}{*}{$\begin{array}{l}\text { Obesidad } \\
230 \mathrm{~kg} / \mathrm{m}^{3} \\
(n=45)\end{array}$} & \multirow[t]{2}{*}{ EOAD } & $r$ & 1 & 0.117 & -0.269 & 0.136 & $0.346^{\circ}$ & 0.225 \\
\hline & & $D$ & & 0.466 & 0.074 & 0.374 & 0.080 & 0.138 \\
\hline & \multirow{2}{*}{$\begin{array}{l}\text { PORCENTAJE } \\
\text { GRASA (\$) }\end{array}$} & $r$ & 0.117 & 1 & $0.711^{\cdots}$ & $0.637^{* 1}$ & $0.45 T^{* 1}$ & -0.220 \\
\hline & & $p$ & 0.446 & & 0.000 & 0.000 & 0.002 & 0.147 \\
\hline & \multirow{2}{*}{$\begin{array}{l}\text { PORCENTAJE DE } \\
\text { MÚSCULO }(\$)\end{array}$} & $t$ & -0.269 & $-0.711 *$ & 1 & $-0.588^{* *}$ & $0.586^{4 *}$ & 0.243 \\
\hline & & $p$ & 0.074 & 0.000 & & 0.000 & 0,000 & 0.100 \\
\hline & \multirow{2}{*}{$\begin{array}{l}\text { CIRCUNFERENCIA } \\
\text { CINTURA }(\mathrm{cm})\end{array}$} & $f$ & 0.138 & $0.637^{* *}$ & $0.588^{\circ *}$ & $t$ & $0.488^{* 4}$ & $-0.309^{* *}$ \\
\hline & & $D$ & 0.374 & 0.000 & 0.000 & & 0.001 & 0.007 \\
\hline & \multirow{2}{*}{$\begin{array}{l}\text { SALTO RELATIVO } \\
\text { (em/kg) }\end{array}$} & $t$ & $-0.346^{\circ}$ & $0.457^{*}$ & $0.585^{4}$ & $0.488^{* *}$ & 1 & 0.187 \\
\hline & & s. & 0.020 & 0.002 & 0.000 & 0.001 & & 0.218 \\
\hline & \multirow[t]{2}{*}{ WOOL (puntajo) } & $r$ & 0.225 & 0220 & 0248 & $0.399=$ & 0.187 & 1 \\
\hline & & s. & 0.139 & 0.147 & 0.100 & 0.007 & 0.218 & \\
\hline
\end{tabular}


En la tabla 3 se detalla la correlación de variables ajustada para grupos de IMC (sobrepeso y obesidad), en el grupo de sobrepeso la edad mantiene una relación negativa con el salto relativo y pierde la relación con el porcentaje de grasa, porcentaje de músculo, circunferencia de cintura y el puntaje total del cuestionario IWQOL. El porcentaje de grasa mantiene una relación negativa con el porcentaje de músculo, pero se pierde la relación con la circunferencia de cintura, salto relativo y el puntaje total del cuestionario IWQOL. El porcentaje de músculo mantiene una relación positiva con el salto relativo y mantiene una relación negativa con la circunferencia de cintura, pero pierde la relación con el puntaje total del cuestionario IWQOL. La circunferencia de cintura mantiene una relación negativa con la variable salto relativo y el puntaje total del cuestionario IWQOL. En el grupo de sobrepeso el salto relativo pierde su relación con el puntaje total del cuestionario IWQOL.

En el grupo de obesidad la edad mantiene una relación negativa con la variable salto relativo, pero se pierde la relación con el porcentaje de grasa, porcentaje de músculo, circunferencia de cintura y el puntaje total del cuestionario IWQOL. El porcentaje de grasa mantiene una relación positiva con la circunferencia de cintura y una relación negativa con el porcentaje de músculo y la variable salto relativo, pero se pierde la relación con el puntaje total del cuestionario IWQOL. La circunferencia de cintura mantiene una relación negativa con la variable salto relativo y el puntaje total del cuestionario IWQOL. En el grupo de obesidad se pierde la relación ente el salto relativo y el puntaje total del cuestionario IWQOL.

\section{Discusión}

Los resultados encontrados en la presente investigación demostraron que la fuerza muscular se relaciona positivamente con la calidad de vida (a mayor fuerza muscular mayor calidad de vida). Según Gustavo Contreras y colaboradores (2011) al analizar la eficacia de un programa de entrenamiento de fuerza y resistencia muscular, con la finalidad de mejorar la capacidad funcional y calidad de vida en paciente renales, demostró que los participantes que demostraron mayor fuerza muscular presentaban mejor cálida de vida evidenciado en el dominio físico de la calidad de vida. Para medir la calidad de vida utilizaron KDQOL-SF un cuestionario aplicado a pacientes con enfermedades del riñón, donde comprende dos rubros físico y mental. (Anthony J, 2010). De igual manera, se ha demostrado una mejoría significativa en la fuerza-resistencia gracias a un programa de acondicionamiento físico intradiálisis en paciente renales y un aumento en el puntaje de calidad de vida medido con el instrumento KDQOL-SF. (González, 2017). 
El estudio coincide con la presente investigación demostrando que la fuerza muscular medida mediante el salto vertical tiene una relación positiva con los rubros del cuestionario IWQOL: actividades de la vida diaria y actividad física. Considerando que el rubro de actividades de la vida diaria incluyó preguntas como dificultad en encontrar ropa con la talla adecuada, para usar las puertas giratorias del tren subterráneo, o para sentarse en las butacas de los cines, estos rubros se enfocan en la funcionalidad del paciente y en el impacto de su peso para realizar actividades físicas. Mazón y colaboradores, (2018) en su estudio concluyen que a medida que se acondiciona físicamente al paciente (adulto mayor), el puntaje de la calidad de vida medida con el instrumento IWQOL aumenta.

$\mathrm{Al}$ relacionar la edad con el IMC se encontró una relación negativa (a mayor sea la edad menor será el IMC). La edad también se relaciona negativamente con el salto relativo (a mayor sea la edad menor será la fuerza muscular evidenciada por salto vertical).

Existen varios factores que reducen la fuerza muscular, principalmente el proceso de envejecimiento, la actividad física disminuye con la edad y aumentan los comportamientos sedentarios. (Poggiogalle E, 2013).

El IMC también tiene una relación negativa con el salto relativo (a mayor sea el IMC menor será la fuerza muscular evidenciada por el salto vertical). Según Crespo y colaboradores (1996) las personas con obesidad presentan disminución en su fuerza muscular de las extremidades inferiores y experimentaron una disminución más pronunciada en la velocidad al caminar siendo más vulnerables en sufrir algún tipo de discapacidad en comparación con personas con un IMC $\leq 25 \mathrm{~kg} / \mathrm{m}^{2}$, para evidenciar la disminución en la fuerza muscular en las extremidades inferiores utilizaron Chair stand test y Brisk Walking test, esta afección fue más evidente en mujeres mayores de 70 años, (American College of Sports Medicine, 2010), al comparar con la presente investigación la media de edad fue $40.96 \pm 11.97$ y se evidencia una disminución en la fuerza muscular con la progresión del IMC.

\section{Conclusión}

Los resultados han demostrado una relación positiva cumpliendo la hipótesis, a mayor sea el salto vertical mayor será la calidad de vida. Un hallazgo importante en la presente investigación es la relación negativa entre los indicadores diagnósticos de obesidad (IMC, Porcentaje de grasa y circunferencia de cintura) con la fuerza muscular y la calidad de vida, mientras que la masa muscular mantiene una relación positiva con la fuerza muscular determinada mediante el salto vertical y la calidad de vida evidenciada mediante el cuestionario IWQOL. 


\section{References:}

1. Alhambra, D., Premaor, M.O., Avilés, F., Hermosilla, E., Martinez, D., Carbonell, C. (2012). The association between fracture and obesity is site dependent: a population-based study in postmenopausal women. Bone Mineral. 27(2): 294-300.

2. American College of Sports Medicine. (1998). Position Stand. Exercise and physical activity for older adults. Medicine Sciences of Sports and Exercise. 30(6): 992-1008.

3. American College of Sports Medicine. (2010). ACSM's health-related physical fitness assessment manual. Philadelphia: Lippincott Williams \& Wilkins.

4. Anthony, J., Graham, E., Mercer, G. (2010). Total Knee Replacement In The Morbidly Obese: A Literature Reviewans. Journal Of Surgery. 80(9); 594-599.

5. Barrios Ospino, Y., Díaz, N., Meertens, L., Naddaf, G., Solano, L., Fernandez, M., Flores, A., González, M. (2010). Relation between leptin serun with weight and body fat distribution in postmenopausal women. Nutricion Hospitalaria. 25:80-84.

6. Bolado, V., García, J., López, A., González, J., Barrancod, A., Comuzzie, G. (2008). Reproducibilidad y sensibilidad del cuestionario "Impacto del peso en la calidad de vida" en mexicanos obesos. Gaceta Medica de México. 155(5):419-425.

7. Cámara, J. (2011). Análisis de la marcha: sus fases y variables espacios temporales. 13:160-173.

8. Contreras, G., Delgado, M., Martínez, J., Parra, I., Borrego, F., Segura, P. (2011). Eficacia de un programa de entrenamiento intradiálisis de fuerza-resistencia en combinación con electroestimulación neuromuscular: Mejora en la capacidad funcional, fuerza y calidad de vida. 14(2):112-119

9. Crespo, C.J., et al. (1996). Leisure-time physical activity among US adults. Results from the Third National Health and Nutrition Examination Survey. Journal of the American Medical Association. 156(1): 93-98.

10. Dakin, S., Dudhia, J., Smith, R. (2014). Resolving an inflamatory concept: the importance of inflammation and resolution in tendinopathy, Immunology and Immunopathology. (158) 3-4,121127.

11. De Laet, C., Kanis, J.A., Odén, A., Johanson, H., Johnell, O., Delmas, P. (2005). Body mass index as a predictor of fracture risk: A Metaanalysis. Osteoporos Int. 16:1330-1338. 
12. Encuesta Nacional de Salud y Nutrición. ENSANUT. (2012). Resultados Nacionales Nutrición 2012. Sobrepeso y obesidad en adultos. 180-186.

13. Feldman, H., Longcope, C., Derby, C., Johannes, C., Araujo, A., Coviello, A. (2002). Age trends in the level of serum testosterone and other hormones in middle-aged men: longitudinal results from the Massachusetts male aging study. Journal Clinical Endocrinology Metabolism. 87:589-598.

14. Franceschi, F., Papalia, R., Paciotti, M., Maffulli, N. (2014). Obesity as a risk factor for tendinopathy: A Systematic Review. 10:1155.

15. Fredman, S., Milner, C., Thompson, D., Zhao, X. (2012). The influence of body mass index and velocity on knee biomechanics during walking. 966- 6362.

16. Garawi, F., Devries, K., Thorogood, N., Ruauy, R. (2014). Differences between women and men in the prevalence of obesity across countries: is there an association with gender inequality. 1-5.

17. Gonnelli, E., Caffarreli, C., Nuti, R. (2014). Obesity and Fracture risk. 11(1): 9-14.

18. González, I., Vega, G. (2017). Fisioterapia Nefrológica: Mejora De La Calidad De Vida Mediante Un Programa De Acondicionamiento Físico En Pacientes Con Enfermedad Renal Crónica En Una Unidad De Hemodiálisis En México. European Scientific Journal. 13(24):405 $-415$.

19. León, H.H., Melo, C.E., Ramírez, J.F. (2012). Role of the myokines production through the exercise. Journal of Sport and Health Research. 4(2):157-166.

20. Lovejoy, J.C. (2003). The menopause and obesity. Primary Care: Clinics in Office Practice. 30:317-325.

21. Mazón, O., Lomas, P., Fernández, D., Santillan, R., Palacios, D. (2018). Calidad De Vida Y El Equilibrio Dinámico En El Adulto Mayor. European Scientific Journal. 14(14):110 - 120.

22. Michele, A., Schiavone, C., Vincenzo, S. (2012). Achilles tendon and plantar fascia in recently diagnosed type II diabetes: role of body mass index. 31:1109-1113.

23. Nahdiya Zainal, A., Mohd Bakri, A. (2013). Prediction of Vertical Jump Height from Anthropometric Factors in Male and Female Martial Arts Athletes. Malaysian Journal of Medical Sciences. 20(1): 39-45.

24. National Institute of Health (NIH). (2001). Consensus development panel on osteoporosis prevention, diagnosis and therapy. JAMA. 285: 785-795. 
25. Navara, H., Cortes, P., Garcia, A. (2011). Papel del adipocito en la expresión del factor inducible por hipoxia (HIF) asociado a la obesidad. Revista 70(4):261-266.

26. Organización Mundial de la Salud. (OMS). (2005). Physical Status: The use and interpretation of anthropometric physical status. Report of a WHO Expert committee.

27. Peña, H. (2007). Prevalencia y factores de riesgo de la osteoartritis. Reumatología Clínica. 3(3):6-12.

28. Picavet, H., Schouten, J.S. (2003). Musculoskeletal pain in the Netherlands: prevalences, consequences and risk groups, the DMC (3)study. 102:167-178.

29. Poggiogalle, E., Mosca, V., Pinto, A., Brunani, A., Capodaglio, P. (2013). Disability affects the 6-minute walking distance in obese subjects. Publication of peer-reviewed science. 8(10); 75491.

30. Reyna, J., Martínez, S., Pascual, M., Rabino, C. (2013). Magnoterapia y ejercicio físico para el tratamiento de la lumbalgia. 1-116.

31. Tommaselli, G.A., Di Carlo, C., Pellicano, M., Nasti, A., Ferrara, C., Di Spiezio Sardo, A., et al. (2001). Modificazioni dei livelli sierici di leptina in menopausa. Minerva Ginecológica. 53:193-198.

32. William, G., Fruhbeck, G. (2009). Obesity Science to Practice. Regulation of energy intake and expenditure. $118-120$. 